Published in final edited form as:

Genet Med. 2021 July ; 23(7): 1288-1295. doi:10.1038/s41436-021-01125-w.

\title{
Variant Curation Expert Panel Recommendations for RYR1 Pathogenicity Classifications in Malignant Hyperthermia Susceptibility
}

\author{
Jennifer J. Johnston, $\mathrm{PhD}^{1}$, Robert T. Dirksen, $\mathrm{PhD}^{2}$, Thierry Girard, $\mathrm{MD}^{3}$, Stephen G. \\ Gonsalves, PhD ${ }^{1}$, Phil M. Hopkins, MD ${ }^{4}$, Sheila Riazi, MD ${ }^{5}$, Louis A. Saddic III, MD ${ }^{6}$, \\ Nyamkhishig Sambuughin, $\mathbf{P h D}^{7}$, Richa Saxena, $\mathbf{P h D}^{8}$, Kathryn Stowell, $\mathbf{P h D}^{9}$, James \\ Weber, PhD ${ }^{10}$, Henry Rosenberg, MD ${ }^{11}$, Leslie G. Biesecker, MD ${ }^{1,12}$
}

\begin{abstract}
${ }^{1}$ Medical Genomics and Metabolic Genetics Branch, National Human Genome Research Institute, National Institutes of Health, Bethesda, MD ${ }^{2}$ Department of Pharmacology and Physiology, University of Rochester Medical School, Rochester, NY ${ }^{3}$ Department of Anesthesiology, University of Basel, Basel, Switzerland ${ }^{4} \mathrm{MH}$ Unit, Leeds Institute of Medical Research at St James's, University of Leeds, Leeds, UK ${ }^{5}$ Department of Anesthesia and Pain Medicine,University Health Network, University of Toronto, Toronto, Canada ${ }^{6}$ Department of Anesthesiology, University of California Los Angeles, Los Angeles, CA ${ }^{7}$ Consortium for Health and Military Performance, Uniformed Services University Health Science, Bethesda, MD ${ }^{8}$ Department of Anesthesia, Critical Care and Pain Medicine, Massachusetts General Hospital, Harvard Medical School, Boston, MA ${ }^{9}$ School of Fundamental Sciences, Massey University, Palmerston North, New Zealand ${ }^{10}$ Prevention Genetics, Marshfield, WI ${ }^{11} \mathrm{MH}$ Association of the United States, Sherburne, NY ${ }^{12} \mathrm{NIH}$ Intramural Sequencing Center, National Human Genome Research Institute, National Institutes of Health, Bethesda, MD
\end{abstract}

\begin{abstract}
Purpose-As a ClinGen Expert Panel (EP) we set out to adapt the ACMG pathogenicity criteria for classification of $R Y R 1$ variants as related to autosomal dominantly-inherited malignant hyperthermia $(\mathrm{MH})$.
\end{abstract}

Methods-We specified ACMG/AMP criteria for variant classification for RYR1 and MH. Proposed rules were piloted on 84 variants. We applied quantitative evidence calibration for several criteria using likelihood ratios based on the Bayesian framework.

\footnotetext{
Users may view, print, copy, and download text and data-mine the content in such documents, for the purposes of academic research, subject always to the full Conditions of use:http://www.nature.com/authors/editorial_policies/license.html\#terms

Corresponding Author: Jennifer J Johnston, Phone: 301-594-3981, jjohnsto@mail.nih.gov.

AUTHOR CONTRIBUTION

Conceptualization: L.G.B.; Data curation: J.J.J., S.G.G., K.S., L.G.B.; Formal Analysis: J.J.J., L.G.B.; Methodology: J.J.J., R.T.D., T.G., S.G.G., P.M.H., S.R., L.A.S., N.S., R.S., K.S., J.W., N.R., L.G.B.; Project Administration: J.J.J., L.G.B.; Writing-original draft: J.J.J., L.G.B.; Writing-review \& editing: R.T.D., T.G., S.G.G., P.M.H., S.R., L.A.S., N.S., R.S., K.S., J.W., H.R.

Conflict of Interest:

LGB has received in kind research support from ArQule, Inc (now wholly owned by Merck, Inc) and honoraria from Cold Spring Harbor Press.
} 
Results-Seven ACMG/AMP criteria were adopted without changes, nine were adopted with $R Y R 1$-specific modifications, and ten were dropped. The in silico (PP3 and BP4) and hot spot criteria (PM1) were evaluated quantitatively. REVEL gave an odds ratio (OR) of 23:1 for PP3 and 14:1 for BP4 using trichotomized cut-offs of $\searrow 0.85$ (pathogenic) and $₫ 0.5$ (benign). The PM1 hotspot criterion had an OR of 24:1. PP3 and PM1 were implemented at moderate strength. Applying the revised ACMG criteria to 44 recognized MH variants, 29 were classified as pathogenic, 13 as likely pathogenic, and two as variants of uncertain significance.

Conclusion-Curation of these variants will facilitate classification of $R Y R 1 / \mathrm{MH}$ genomic testing results, which is especially important for secondary findings analyses. Our approach to quantitatively calibrating criteria is generalizable to other variant curation expert panels.

\section{INTRODUCTION}

Malignant hyperthermia susceptibility (MHS) is a potentially lethal inherited disorder of skeletal muscle calcium signaling, predisposing individuals to a hypermetabolic reaction triggered by exposure to inhalational anesthetics or depolarizing muscle relaxants such as succinylcholine. ${ }^{1,2}$ Inheritance of MHS is predominantly autosomal dominant, although autosomal recessive inheritance has been reported ${ }^{3}$ and non-Mendelian models proposed. ${ }^{4}$ Variants in RYR1 (MIM:180901; MHS1, MIM:145600) and CACNA1S (MIM:114208; MHS5, MIM:601887) have been associated with MH, and both genes are in the American College of Medical Genetics and Genomics (ACMG) return of secondary findings recommendations. ${ }^{5,6} R Y R 1$ variants account for $\sim 76 \%$ of $\mathrm{MH}$ events while $\sim 1 \%^{7}$ are attributable to $C A C N A 1 S$ and $<1 \%$ are attributable to $S T A C 3$ (MIM:615521; Bailey-Bloch myopathy, MIM:255995). Four additional loci have been mapped (MHS2, MIM:154275; MHS3, MIM:154276; MHS4, MIM:600467; MHS6, MIM:601888). RYR1 has a complex gene-to-phenotype relationship, being associated with several apparently distinct disorders and both autosomal dominant and autosomal recessive inheritance. Overlapping conditions include central core disease (CCD, MIM:117000) and King-Denborough syndrome (MIM:145600) and individuals with these disorders may be at risk for MH. Generally, these disorders result from monoallelic $R Y R 1$ variants while biallelic variants cause other myopathies, however, this correlation is evolving. ${ }^{8}$

Classification of $R Y R 1$ variants is complicated by variable expressivity, reduced penetrance, and high alleleic heterogeneity. While the European Malignant Hyperthermia Group (EMHG; http://www.emhg.org/home/) has assessed 48 RYR1 variants as diagnostic of MHS, over 165 additional variants have been reported as disease mutations/pathogenic/ likely pathogenic for $\mathrm{MH}$ in the literature and databases including $\mathrm{HGMD}^{9}$ and ClinVar. ${ }^{10}$ While the ACMG/AMP guidelines provided general criteria that can be used to classify variants, many of the criteria require adaptation to be accurately applied. As part of ClinGen, we convened an $R Y R$ 1-related Malignant Hyperthermia variant curation expert panel (https://clinicalgenome.org/affiliation/50038/) to adapt the general ACMG/AMP pathogenicity guidelines to autosomal dominantly inherited $R Y R 1 \mathrm{MH}$, with gene-specific recommendations, to improve classification of $R Y R 1$ variant pathogenicity. 
We first reviewed each ACMG/AMP criterion to determine applicability to autosomal dominantly inherited $R Y R 1 \mathrm{MH}$ and then adapted them with gene/disease specific guidelines, if appropriate. We piloted these guidelines on 84 variants, 44 variants from the EMHG list of "diagnostic mutations" and 40 variants with MH pathogenicity classifications in ClinVar.

\section{METHODS}

\section{ClinGen's RYR1/MH Expert Panel}

The RYR1MH expert panel (EP) is composed of clinical molecular geneticists, clinical geneticists, anesthesiologists, biochemists, and physiologists to provide a balance of expertise relevant to $R Y R 1$ variant classification. The $R Y R 1 / \mathrm{MH}$ EP met monthly via conference calls over a two-year period.

\section{Evaluation and Adaptation of the ACMG Pathogenicity Guidelines}

The general ACMG/AMP pathogenicity guidelines were evaluated for relevance to autosomal dominantly-inherited $R Y R 1 \mathrm{MH}$ and non-relevant criteria were dropped. ClinGen-recommended amendments to the criteria were incorporated when applicable. Lastly, applicable criteria were further assessed to determine if gene-specific recommendations were warranted. Proposed changes were discussed amongst the full EP by emails and conference calls. Approval of revised rules required consensus of the full EP. Draft rules were piloted on a subset of $R Y R 1$ variants representing the EMHG "diagnostic mutation" list. Individual panel members scored variants using the draft guidelines and variant classifications were presented to the full panel. Areas of disagreement were used to refine the draft guidelines. Per the ClinGen FDA-approved process, rules were reviewed by the ClinGen Sequence Variant Interpretation (SVI) committee (LGB recused).

\section{Data Collection Methods}

Population data was ascertained from gnomAD v2.1.1. ${ }^{11}$ REVEL scores (v0.19.1) were used for bioinformatic predictions for single nucleotide variants (SNVs). ${ }^{12}$ The literature was searched for relevant data including case information and functional data. For case information, the number of unrelated probands with either a personal or family history of an MH event was recorded (see supplemental information). Care was taken to avoid double counting cases reported multiple times. Reports were examined for instances of de novo inheritance and/or segregation.

\section{Pathogenicity Assessment}

Revised ACMG/AMP criteria were used to assess 44 EMHG MH "diagnostic mutations". Four of 48 EMHG variants were excluded because they were associated with $R Y R 1$-related myopathies and not MH. An additional 40 ClinVar $R Y R 1$ variants were also classified. Individual criteria were weighted based on available evidence and weighted criteria were combined using the Bayesian framework for variant scoring. ${ }^{13}$ 


\section{RESULTS AND DISCUSSION}

The ACMG/AMP guidelines are generic and broadly useful for all Mendelian genes and disorders. These generic rules may over- or under-estimate evidence for any specific gene and must be adapted for specific implementations. As an EP, we suggest guidelines to be used/dropped, guidelines to be refined, and weight adjustments where appropriate. A summary of revised guidelines is in Table 1 and a full description is in Table S1 with gene/ disease specific adaptations highlighted below (updated versions will be maintained at clinicalgenome.org).

\section{Criteria Dropped for MH/RYR1: PVS1/PM2/PM3/PM4/PP2/PP4/BS4/BP1/BP3/BP5}

These criteria were dropped based on the biology of MH/RYR1. See supplemental information for details.

\section{Criteria Used According to General Guidelines: PS1/PS2/PM5/PM6/PP1/BP2/BP7}

These criteria were retained in the $R Y R 1 / \mathrm{MH}$-specific guidelines including adaptations as recommended by the ClinGen SVI committee (PS2/PM6, weighting of de novo observations, https://clinicalgenome.org/site/assets/files/3461/ svi_proposal_for_de_novo_criteria_v1_0.pdf) and the Cardiomyopathy EP (PP1, weighting segregation events). ${ }^{14}$ We made further modifications to the ACMG/AMP criteria, which may not be specific to $R Y R 1 / \mathrm{MH}$. The PS1 (same amino acid change, different nucleotide change) and PM5 (different amino acid change, same codon) criteria were modified such that in order to use either of them, a previously classified variant must have been classified as pathogenic without the use of PS1 or PM5. Furthermore, for PM5, we added a requirement that the Grantham score difference compared to reference of the new variant must be greater than that for the previously identified pathogenic variant compared to reference. For criterion BP2 (evidence against pathogenicity based on presence of known pathogenic variant) it is suggested that only variants identified in cis with the variant under review be considered. Because the occurrence of biallelic pathogenic $R Y R 1$ variants has been described in MHS, ${ }^{3,15}$ two variants in trans is not considered evidence against pathogenicity. Finally, as $R Y R 1 / \mathrm{MH}$ primarily results from missense alterations, BP7 (synonymous variant without splicing effect) is used as recommended.

\section{Criteria Specified for RYR1/MH: BA1/BS1/PS4/BS2/PS3/BS3/PM1/PP3/BP4}

Allele Frequency Specificiations: BA1/BS1/PS4-BA1 and BS1 use minor allele frequencies (MAF) in population datasets to support benign classification for common variants. The BA1 criterion is considered stand alone and was originally set to $0.05(5 \%)$ MAF. ${ }^{16}$ It has been suggested that BA1 can be defined as the combined MAF for all pathogenic variants in the population for the gene/disease dyad with the understanding that any one variant should have a lower MAF than the combined total. To determine a gene/ disease-specific cutoff for BA1, disease prevalence, penetrance, and gene contribution need to be considered. This can be estimated by the formula: $\left(\frac{\text { [disease prevalence] } x[\% \text { gene contribution] }}{\text { [penetrance] }}\right) .{ }^{14}$ The prevalence of $\mathrm{MH}$ (defining the disorder as $\mathrm{MH}$, not MHS) in the population can be estimated using the frequency of $\mathrm{MH}$ events in 
individuals exposed to triggering agents. The frequency of events is as high as $1 / 10,000$ pediatric anesthesias. ${ }^{2}$ The rate of adult $\mathrm{MH}$ events seems lower than that of children ${ }^{17}$ but the underlying genetic risk is assumed to be the same. The gene contribution of $R Y R 1$ to $\mathrm{MH}$ is $\sim 76 \%$ depending on ethnicity. ${ }^{7}$ Calculating thresholds for BA1 relies on an accurate estimate of penetrance, which is difficult to determine for MHS. ${ }^{18}$ In lieu of using an estimate for MHS penetrance, we instead substituted a value of $1 \%$, as it is a reasonable boundary between the penetrance of a Mendelian disorder variant and that of a risk allele. This value is nearly certain to be lower than the actual penetrance of MHS, but underestimating this value is conservative with respect to the outcome in that it will numerically raise BA1, which would lead to fewer variants being classified as benign based on this single criterion. Using 0.01 to adjust our calculated BA1 allows for a BA1 MAF of $0.0038(0.38 \%)$.

In addition to a stand alone MAF (BA1), BS1 defines the MAF at which a variant is considered to have strong evidence against pathogenicity. The field has been moving to define BS1 based on the contribution of the most common pathogenic allele for a disorder. For $R Y R 1 / \mathrm{MH}$, we calculated BS1 considering the frequency of $\mathrm{MH}$ reactions in children $(1 / 10,000)$ a value of 0.01 substituted for penetrance (as explained above), and a maximum individual allele contribution of $16 \%$ (variant c.7300G > A was identified in 118/722 MH families, $16.3 \%) .{ }^{7}$ Correcting for alleles/person gives a BS1 value of $0.0008(0.08 \%)$.

While a high MAF of a variant in controls can be used to refute pathogenicity, criterion PM2 gives weight for absence or very low frequency in control populations. Based on observations that the majority of possible $R Y R 1$ missense variants ( 30,000 variants) are not represented in gnomAD v2.1.1 (2,800 RYR1 missense variants) and many known pathogenic variants (classified without the use of PM2) are present in gnomAD, it is unlikely that the absence of a variant in gnomAD is support for pathogenicity. While the absence or low frequency of a variant in gnomAD has little value alone, it is important in weighting PS4. PS4 takes into consideration the prevalence of the variant in affected individuals compared to controls. For $R Y R 1 / \mathrm{MH}$, we modified the PS4 criterion using a point system, awarding 0.5 case points for each unrelated proband reported to have undergone an $\mathrm{MH}$ event and awarding an additional 0.5 case points for a positive in vitro contracture test (IVCT) or caffeine-halothane contracture test (CHCT) in either the proband or a variantpositive family member. The strength level of PS4 is based on odds ratios comparing total case points, an approximation of the total number of cases of $\mathrm{MH}$ investigated in the literature $(3,000)$ and the number of alleles in the gnomAD continental population with the highest MAF (popmax). When the popmax MAF is $\$ 0.00006$ ( 7/113,000 alleles), strength levels are awarded according to the following system: PS4 for $\geq 7 \mathrm{MH}$ case points; PS4_Mod for 2-6 MH case points; and PS4_Sup for one MH case point. When gnomAD popmax MAF is $>0.00006$, case points can be counted and compared to alleles in the gnomAD population with the highest MAF by calculating an odds ratio (OR, MedCalcs online calculator (https:// www.medcalc.org/calc/odds_ratio.php), awarding PS4 for an OR $\geq 18.7$; PS4_Mod for an OR 24.33 ; and PS4_Sup for an OR $\geq 2.08$. Every effort needs to be made to avoid double counting of cases reported in multiple studies. The Bayesian framework for the classification of variants using the ACMG/AMP criteria was used to set the OR value for each strength level. ${ }^{13}$ 
Disease-Specific Phenotype: BS2-The IVCT/CHCT diagnostic tests have low false negative rates ${ }^{19,20}$ and can be used to determine MHS status in individuals who carry $R Y R 1$ variants. A negative IVCT or CHCT result supports benign status. Two or more unrelated individuals with a negative result allow BS2 to be applied. One individual with a negative result allows BS2_Mod.

Functional Assay Specifications: PS3/BS3-Functional characterization is considered a crucial determinant of the pathogenicity of $R Y R 1$ variants. ${ }^{21}$ Within the ACMG/AMP guidelines, functional assay results are used for PS3 (well-established in vitro or in vivo functional studies supportive of a damaging effect) and BS3 (well-established in vitro or in vivo functional studies show no damaging effect on protein function or splicing). RYR1 is a homotetrameric calcium channel in the sarcoplasmic reticulum (SR) of skeletal muscle important in excitation-contraction coupling. Volatile anesthetics and depolarizing muscle relaxants can cause increased release of SR calcium in a dysfunctional RYR1 channel resulting in $\mathrm{MH}$. When considering functional assays for variant assessment it is desirable to identify assays that are closely related to the physiologic defect causative of disease. For $R Y R 1 / \mathrm{MH}$, assays that measure release of calcium in response to pharmacologic agents are considered good representations of the disease mechanism. Wellrecognized assays include transfection of $R Y R 1$ cDNA into HEK293 cells, CHO cells, or $R Y R 1$ knockout myotubes followed by SR calcium release measurements in response to caffeine, halothane, voltage/potassium, or 4-chloro- $m$-cresol. A significant decrease in the $\mathrm{EC}_{50}$ for the sensitivity of calcium release compared to wildtype RYR1, is considered evidence for pathogenicity. Multiple replicates for each variant within a single instance of the assay are necessary to determine significance of these values. Positive (pathogenic) and negative (benign) controls support that the assay categorizes the variants accurately. For the purpose of assessing $R Y R 1$ transfection studies to weight PS3, results were dichotomized into pathogenic $\mathrm{EC}_{50}$ values that are significantly decreased as compared to WT versus benign $\mathrm{EC}_{50}$ values that are not significantly decreased. For $R Y R 1$ pathogenicity assessment, the whole of prior published work (Figure 1, Table S2) ${ }^{22}$ allows us to consider transfection assays in HEK293 cells using photometry/imaging to measure calcium release a well defined functional test. However, recommendations for increased stringency in analyses of functional data have recently been suggested. ${ }^{23}$ To determine the appropriate PS3 weight based on HEK293 transfection assays we have considered published results including results for 35 variants assessed to be likely pathogenic or pathogenic (LP/P) without the use of functional data, and ten control variants including eight variants associated with $\mathrm{CCD}$ and two common variants. Of the $35 \mathrm{LP} / \mathrm{P}$ variants, 29 have been shown to reduce the calcium release $\mathrm{EC}_{50}$ in response to RYR1 agonsits. Five variants have shown discordant results across assays, and one variant has shown an $\mathrm{EC}_{50}$ increase. Of the ten control variants, one variant has shown an $\mathrm{EC}_{50}$ reduction in response to agonist and nine variants have either shown no response to agonist (6) or a response similar to WT (3). This set of variants suggests a likelihood ratio for an $\mathrm{EC}_{50}$ reduction of 9.11:1 with a $95 \%$ confidence interval of 1.4:1 to 59:1. This level of support is above the threshold for moderate evidence (4.33:1 odds). We suggest that functional evidence supporting pathogenicity from HEK293 cells be used at the level of moderate. When the field generates additional data for control variants the weighting of PS3 for this assay should be reconsidered. 
While positive evidence (reduced $\mathrm{EC}_{50}$ ) is considered moderate support for pathogenicity, reduced penetrance and the limitations of expression systems, ${ }^{24}$ suggest a non-significant change in $\mathrm{EC}_{50}$ values may not support benign status at a moderate level. It was decided that lack of response to agonists be weighted as supporting evidence, BS3_Sup. Regarding other in vitro assays that test calcium release in response to agonists, where historical data were limited, we suggest that multiple controls be run in parallel and statistical analyses be used to determine the level of strength for PS3 according to the Bayesian framework.

In addition to in vitro assays, the $R Y R 1 / \mathrm{MH}$ field has established ex vivo assays measuring calcium release in patient cells. These assays do not isolate the $R Y R 1$ variant from other potential variants (in $R Y R 1, C A C N A 1 S$, or other MHS-associated genes), which may affect calcium release. Rather, these assays are a measure of the cellular phenotype in the patient. Although we recognize this limitation of ex vivo studies, we also recognize that they have utility. As the main concern for such assays is the potential presence of other variants, this concern is mitigated if multiple unrelated individuals with the same primary variant are shown to exhibit enhanced ex vivo sensitivity to agonist. Two unrelated individuals with $e_{x}$ vivo tests showing increased sensitivity of calcium release in response to agonist allow PS3_Sup. For variants where 23 unrelated individuals had ex vivo tests showing increased sensitivity of calcium release, PS3_Mod can be applied. Ex vivo tests that do not show increased sensitivity of calcium release in response to agonist (negative result) support a benign classification of the variant. BS3_Sup can be applied if one or two unrelated individuals are tested with negative results, when $>3$ unrelated individuals are tested and all results are negative BS3_Mod can be applied.

Knock-in mouse models created to date to test $R Y R 1$ variants have shown MH reactions in response to volatile anesthetic and ex vivo studies of muscle samples from these mice show increased ligand sensitivity of calcium release as compared to WT. ${ }^{25-28}$ When knock-in mice have an $\mathrm{MH}$ reaction in response to agonist, and where ex vivo studies show increased calcium release as compared to WT in response to agonist, PS3 can be awarded. For mouse models where either an $\mathrm{MH}$ crisis can be triggered by agonist or ex vivo assays show increased calcium release, but both conditions are not met, PS3_Mod can be awarded. For mouse models that do not exhibit an MH reaction when exposed to agonist and ex vivo studies do not show increased release of calcium, BS3_Sup can be awarded.

Hotspot Specifications: PM1-The ACMG/AMP criteria includes moderate weight for variation in critical protein domains or mutational hotspots, PM1. While critical domains may be well-defined for a protein, the concept of mutational hotspots is less clearly defined. A general rule for consideration of a mutational hotspot would be an excess of pathogenic variation as compared to benign variation. In $\mathrm{MH}$, variants have been noted to cluster in three regions of $R Y R 1$ identified as "hotspots" historically: the $\mathrm{N}$-terminal region (residues 1-552), the central region (residues 2,101-2,458) and the C-terminal region $(4,631-4,991) .{ }^{29}$ Rather than defining clear functional domains, these regions are defined by an increase in variation identified in individuals with $\mathrm{MH}$. We assessed this criterion using a test set of 19 variants (Table S3) assessed to be pathogenic for MH without the use of PM1 and 27 benign variants (Table S4) that met criterion BA1. This set of variants suggests a likelihood ratio for hotspots of 24:1 with a 95\% confidence interval of 3.4:1 to 163:1 (Table 2). This level of 
support is above the threshold for strong evidence (18.7:1 odds) and the lower bound of that confidence interval is above supporting (2.1:1). This would suggest that PM1 could be modified to PM1_strong. However, because there is a significant bias in the literature toward identifying pathogenic variants in the hotspots, and to avoid the possibility of overestimating pathogenicity, we suggest using PM1 at its default level of moderate for variants in the Nterminal and central regions. As variants in the $\mathrm{C}$-terminal region may be associated with CCD and not cause MH, we suggest using PM1_supporting for variants in this region. Future studies that interrogate the gene without these biases should provide additional data on the positional skewing of pathogenic variants, which could allow upgrading PM1 to strong in the future.

Computational Evidence: PP3/BP4-The PP3 and BP4 criteria consider computational evidence estimating the impact of a variant on protein function. REVEL is an ensemble method based on a number of individual tools and precomputed scores are available for all missense variants (https://omictools.com/revel-tool). ${ }^{12}$ Importantly, REVEL does not consider population frequency, which reduces double counting of evidence. Using a set of 20 pathogenic variants determined to be pathogenic without the use of PP3 and 27 benign variants described above, we tested the likelihood ratios of the predictive power of REVEL in several iterations. We settled on a trichotomization of scores with PP3, (computational evidence supporting pathogenicity), requiring a REVEL score of $\searrow 0.85$ and BP4, (computational evidence against pathogenicity), requiring a REVEL score of $₫ \mathbf{0 . 5}$ (Table 3). Based on the Bayesian model for weighting criteria, these results suggest that PP3 and BP4 could be employed at the strong level. However, based on wide confidence intervals of the likelihood ratios for this conditional probability, we chose to weight PP3 as moderate and BP4 as supporting. ${ }^{13}$ Based on piloting these criteria it was determined that BP4 should only be implemented with other criteria. Using the Bayesian framework, BP4 in isolation results in an assessment of likely benign (LB) and it was determined that additional evidence should be available for a LB classification. For a fuller explanation of deriving such likelihood ratios, see Supplemental information.

Piloting RYR1/MH Classification Criteria-We applied these modified criteria to 44 variants EMHG determined to be "diagnostic mutations" and $40 R Y R 1$ variants with pathogenicity classifications for $\mathrm{MH}$ in ClinVar. The classification of each of the variants is shown in Table S3 and Table S5. Of the 44 EMHG variants, we classified 29 as P, 13 as LP, and two as variants of uncertain significance (VUS). Variant c.1589G >A p.(Arg530His) was classified as VUS and had limited functional data including a single ex vivo sample ${ }^{30}$, which did not meet PS3_Sup based on the requirement for a minimum of two unrelated individuals. Variant c.1598G >A p.(Arg533His) was classified as VUS based on functional data (PS3_Mod) and presence in a hotspot (PM1). PS4 was not met by this variant based on a high allele count (32 alleles) in gnomAD.

The revised criteria were applied to 40 additional variants with pathogenicity classifications for $\mathrm{MH}$ in ClinVar. Ten variants had conflicting pathogenicity classifications for $\mathrm{MH}$ (pathogenicity classifications for disorders other than $\mathrm{MH}$ were not considered), nine $\mathrm{B} / \mathrm{LB} / \mathrm{VUS}$ and one P/LP/VUS. Five variants with B/LB/VUS classifications in ClinVar 
were determined to be $\mathrm{B} / \mathrm{LB}$ based on BA1/BS1. The remaining five discordant variants were classified as VUS. Of the remaining 30 variants, 14 were classified as P/LP, 11 as $\mathrm{B} / \mathrm{LB}$ and five as VUS. Applying the revised ACMG criteria 12/14 variants with a classification of $\mathrm{P} / \mathrm{LP}$ in ClinVar and 3/11 variants with an classification of $\mathrm{B} / \mathrm{LB}$ in ClinVar were classified as VUS. All variants classified as B/LB (13) using our criteria had ether BA1 or BS1 applied. The 19/24 variants classified as VUS had limited data, only five VUS variants had data that refuted pathogenicity $(5 / 24,21 \%)$.

\section{CONCLUSIONS}

As a ClinGen expert panel, we set out to adapt the ACMG pathogenicity criteria for classification of $R Y R 1$ variants as related to autosomal dominanty inherited MH. Combining expertise of anesthesiologists, physiologists, biochemists, and geneticists allowed for a thorough evaluation of factors that should be considered. It is also important to recognize that we successfully unified the efforts of the American-based ACMG/AMP criteria with the extensive expertise and experience of the European Malignant Hyperthermia Group, benefiting from both. In revising these guidelines, we have considered the statistical evidence weight as it relates to the Bayesian adaptation of the ACMG scoring system. Weighting of evidence using statistical measures should allow for a more robust and consistent pathogenicity classification framework and is broadly applicable to other disease/ gene systems. The revised $R Y R 1 / \mathrm{MHS}$ specific criteria should allow clinical laboratories to more consistently classify these variants based on expert guidelines and should increase the consistency of classifications, as has been demonstrated for the generic ACMG/AMP pathogenicity recommendations. ${ }^{31}$ These recommendations should be especially useful to laboratories that classify $R Y R 1$ variants as secondary findings. That $\mathrm{MH}$ is a pharmacogenetic trait with relatively low penetrance makes it especially challenging to classify for laboratories that do not peform a high volume of diagnostic $R Y R 1$ testing. The availability of three star ClinGen classifications in ClinVar should significantly reduce the amount of time that secondary findings evaluations consume. As well, the RYR1/MH expert panel will continue to curate variants and deposit classifications into ClinVar. Moving forward, the field should strive to increase relevant data through functional studies and shared case documentation allowing variants to move from a classification of VUS to either $\mathrm{LB} / \mathrm{B}$ or LP/P. Beyond secondary findings, ClinGen classifications of $R Y R 1$ variant pathogenicity will allow the field to consider pre-surgicial screening of patients toward elimination of MH morbidity and mortality. ${ }^{32}$

\section{Supplementary Material}

Refer to Web version on PubMed Central for supplementary material.

\section{ACKNOWLEDGEMENTS}

ClinGen is funded by the National Human Genome Research Institute: U41HG006834, U41HG009649, U41HG009650. ClinGen receives support from the Eunice Kennedy Shriver National Institute of Child Health and Human Development: U24HD093483, U24HD093486, U24HD093487. The content is solely the responsibility of the authors and does not necessarily represent the official views of the National Institutes of Health. JJJ and LGB were supported by NIH grant HG200359-12. RTD is supported by grant R01 AR053349. PH is supported by the 
National Institute of Arthritis, Musculoskeletal and Skin Diseases: 2P01 AR-05235, 1R01AR068897-01A1. SR is funded by merit award from the Department of Anesthesia and Pain Medicine, University of Toronto, Canada.

\section{DATA AVAILABILITY}

Any variant classification described herein that has not been posted to ClinVar (https:// www.ncbi.nlm.nih.gov/clinvar/?term=ryr1\%5Bgene\%5D) at the time of publication will be made available upon request.

\section{REFERENCES}

1. Gonsalves SG, Dirksen RT, Sangkuhl K et al. Clinical Pharmacogenetics Implementation Consortium (CPIC) guideline for the use of potent volatile anesthetic agents and succinylcholine in the context of RYR1 or CACNA1S genotypes. Clin Pharmacol Ther (2018).

2. Rosenberg H, Sambuughin N, Riazi S \& Dirksen R Malignant Hyperthermia Susceptibility. in GeneReviews((R)) (eds. Adam MP et al.) (University of Washington, Seattle (WA), 1993).

3. Monnier N, Krivosic-Horber R, Payen JF et al. Presence of two different genetic traits in malignant hyperthermia families: implication for genetic analysis, diagnosis, and incidence of malignant hyperthermia susceptibility. Anesthesiology 97, 1067-74 (2002). [PubMed: 12411788]

4. Carpenter D, Robinson RL, Quinnell RJ et al. Genetic variation in RYR1 and malignant hyperthermia phenotypes. Br J Anaesth 103, 538-48 (2009). [PubMed: 19648156]

5. Green RC, Berg JS, Grody WW et al. ACMG recommendations for reporting of incidental findings in clinical exome and genome sequencing. Genet Med 15, 565-74 (2013). [PubMed: 23788249]

6. Kalia SS, Adelman K, Bale SJ et al. Recommendations for reporting of secondary findings in clinical exome and genome sequencing, 2016 update (ACMG SF v2.0): a policy statement of the American College of Medical Genetics and Genomics. Genet Med 19, 249-255 (2017). [PubMed: 27854360]

7. Miller DM, Daly C, Aboelsaod EM et al. Genetic epidemiology of malignant hyperthermia in the UK. Br J Anaesth 121, 944-952 (2018). [PubMed: 30236257]

8. Jungbluth H, Treves S, Zorzato F et al. Congenital myopathies: disorders of excitation-contraction coupling and muscle contraction. Nat Rev Neurol 14, 151-167 (2018). [PubMed: 29391587]

9. Stenson PD, Mort M, Ball EV, Shaw K, Phillips A \& Cooper DN The Human Gene Mutation Database: building a comprehensive mutation repository for clinical and molecular genetics, diagnostic testing and personalized genomic medicine. Hum Genet 133, 1-9 (2014). [PubMed: 24077912]

10. Landrum MJ, Lee JM, Benson $\mathrm{M}$ et al. ClinVar: improving access to variant interpretations and supporting evidence. Nucleic Acids Res 46, D1062-D1067 (2018). [PubMed: 29165669]

11. Karczewski KJ, Francioli LC, Tiao G et al. The mutational constraint spectrum quantified from variation in 141,456 humans. Nature 581, 434-443 (2020). [PubMed: 32461654]

12. Ioannidis NM, Rothstein JH, Pejaver V et al. REVEL: An Ensemble Method for Predicting the Pathogenicity of Rare Missense Variants. Am J Hum Genet 99, 877-885 (2016). [PubMed: 27666373]

13. Tavtigian SV, Greenblatt MS, Harrison SM et al. Modeling the ACMG/AMP variant classification guidelines as a Bayesian classification framework. Genet Med 20, 1054-1060 (2018). [PubMed: 29300386]

14. Kelly MA, Caleshu C, Morales A et al. Adaptation and validation of the ACMG/AMP variant classification framework for MYH7-associated inherited cardiomyopathies: recommendations by ClinGen's Inherited Cardiomyopathy Expert Panel. Genet Med 20, 351-359 (2018). [PubMed: 29300372]

15. Kraeva N, Riazi S, Loke J et al. Ryanodine receptor type 1 gene mutations found in the Canadian malignant hyperthermia population. Can J Anaesth 58, 504-13 (2011). [PubMed: 21455645]

16. Richards S, Aziz N, Bale S et al. Standards and guidelines for the interpretation of sequence variants: a joint consensus recommendation of the American College of Medical Genetics and 
Genomics and the Association for Molecular Pathology. Genet Med 17, 405-24 (2015). [PubMed: 25741868]

17. Halliday NJ Malignant hyperthermia. J Craniofac Surg 14, 800-2 (2003). [PubMed: 14501352]

18. Ibarra Moreno CA, Hu S, Kraeva N et al. An Assessment of Penetrance and Clinical Expression of Malignant Hyperthermia in Individuals Carrying Diagnostic Ryanodine Receptor 1 Gene Mutations. Anesthesiology 131, 983-991 (2019). [PubMed: 31206373]

19. Ording H, Brancadoro V, Cozzolino S et al. In vitro contracture test for diagnosis of malignant hyperthermia following the protocol of the European MH Group: results of testing patients surviving fulminant $\mathrm{MH}$ and unrelated low-risk subjects. The European Malignant Hyperthermia Group. Acta Anaesthesiol Scand 41, 955-66 (1997). [PubMed: 9311391]

20. Allen GC, Larach MG \& Kunselman AR The sensitivity and specificity of the caffeine-halothane contracture test: a report from the North American Malignant Hyperthermia Registry. The North American Malignant Hyperthermia Registry of MHAUS. Anesthesiology 88, 579-88 (1998). [PubMed: 9523799]

21. Hopkins PM, Ruffert H, Snoeck MM et al. European Malignant Hyperthermia Group guidelines for investigation of malignant hyperthermia susceptibility. Br J Anaesth 115, 531-9 (2015). [PubMed: 26188342]

22. Lawal TA, Wires ES, Terry NL, Dowling JJ \& Todd JJ Preclinical model systems of ryanodine receptor 1-related myopathies and malignant hyperthermia: a comprehensive scoping review of works published 1990-2019. Orphanet J Rare Dis 15, 113 (2020). [PubMed: 32381029]

23. Brnich SE, Abou Tayoun AN, Couch FJ et al. Recommendations for application of the functional evidence PS3/BS3 criterion using the ACMG/AMP sequence variant interpretation framework. Genome Med 12, 3 (2019). [PubMed: 31892348]

24. Sato K, Roesl C, Pollock N \& Stowell KM Skeletal muscle ryanodine receptor mutations associated with malignant hyperthermia showed enhanced intensity and sensitivity to triggering drugs when expressed in human embryonic kidney cells. Anesthesiology 119, 111-8 (2013). [PubMed: 23459219]

25. Feng W, Barrientos GC, Cherednichenko G et al. Functional and biochemical properties of ryanodine receptor type 1 channels from heterozygous R163C malignant hyperthermia-susceptible mice. Mol Pharmacol 79, 420-31 (2011). [PubMed: 21156754]

26. Andronache Z, Hamilton SL, Dirksen RT \& Melzer W A retrograde signal from RyR1 alters DHP receptor inactivation and limits window $\mathrm{Ca} 2+$ release in muscle fibers of Y522S RyR1 knock-in mice. Proc Natl Acad Sci U S A 106, 4531-6 (2009). [PubMed: 19246389]

27. Lopez JR, Kaura V, Diggle CP, Hopkins PM \& Allen PD Malignant hyperthermia, environmental heat stress, and intracellular calcium dysregulation in a mouse model expressing the p.G2435R variant of RYR1. Br J Anaesth 121, 953-961 (2018). [PubMed: 30236258]

28. Yuen B, Boncompagni S, Feng W et al. Mice expressing T4826I-RYR1 are viable but exhibit sexand genotype-dependent susceptibility to malignant hyperthermia and muscle damage. FASEB J 26, 1311-22 (2012). [PubMed: 22131268]

29. Maclennan DH \& Zvaritch E Mechanistic models for muscle diseases and disorders originating in the sarcoplasmic reticulum. Biochim Biophys Acta 1813, 948-64 (2011). [PubMed: 21118704]

30. Zullo A, Klingler W, De Sarno C et al. Functional characterization of ryanodine receptor (RYR1) sequence variants using a metabolic assay in immortalized B-lymphocytes. Hum Mutat 30, E57590 (2009). [PubMed: 19191333]

31. Amendola LM, Jarvik GP, Leo MC et al. Performance of ACMG-AMP Variant-Interpretation Guidelines among Nine Laboratories in the Clinical Sequencing Exploratory Research Consortium. Am J Hum Genet 99, 247 (2016). [PubMed: 27392081]

32. Biesecker LG, Dirksen RT, Girard T et al. Genomic Screening for Malignant Hyperthermia Susceptibility. Anesthesiology (2020). 
40

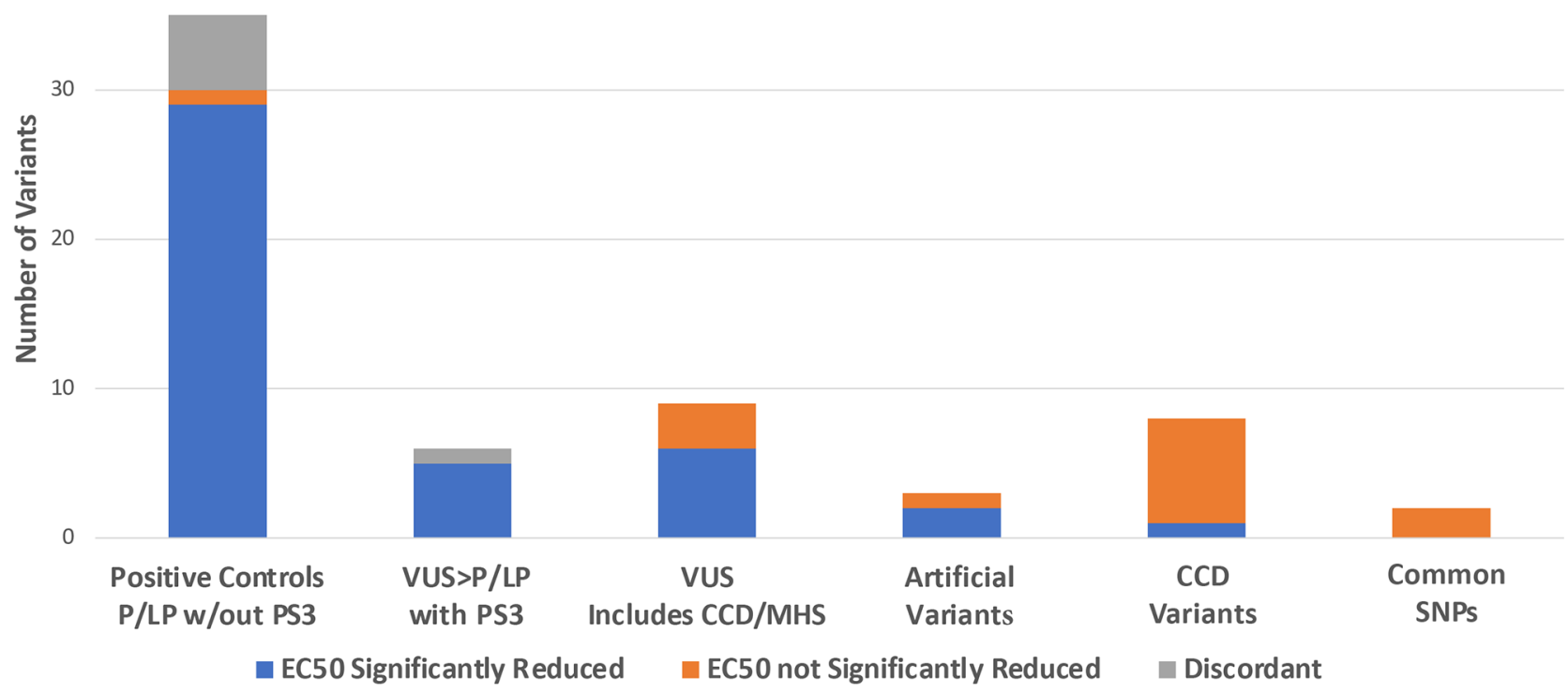

Figure 1.

Cumulative HEK293 transfection assay data for RYR1 variants from the literature. Variants are grouped according to pathogenicity assessment without consideration of PS3/BS3

(functional data). 


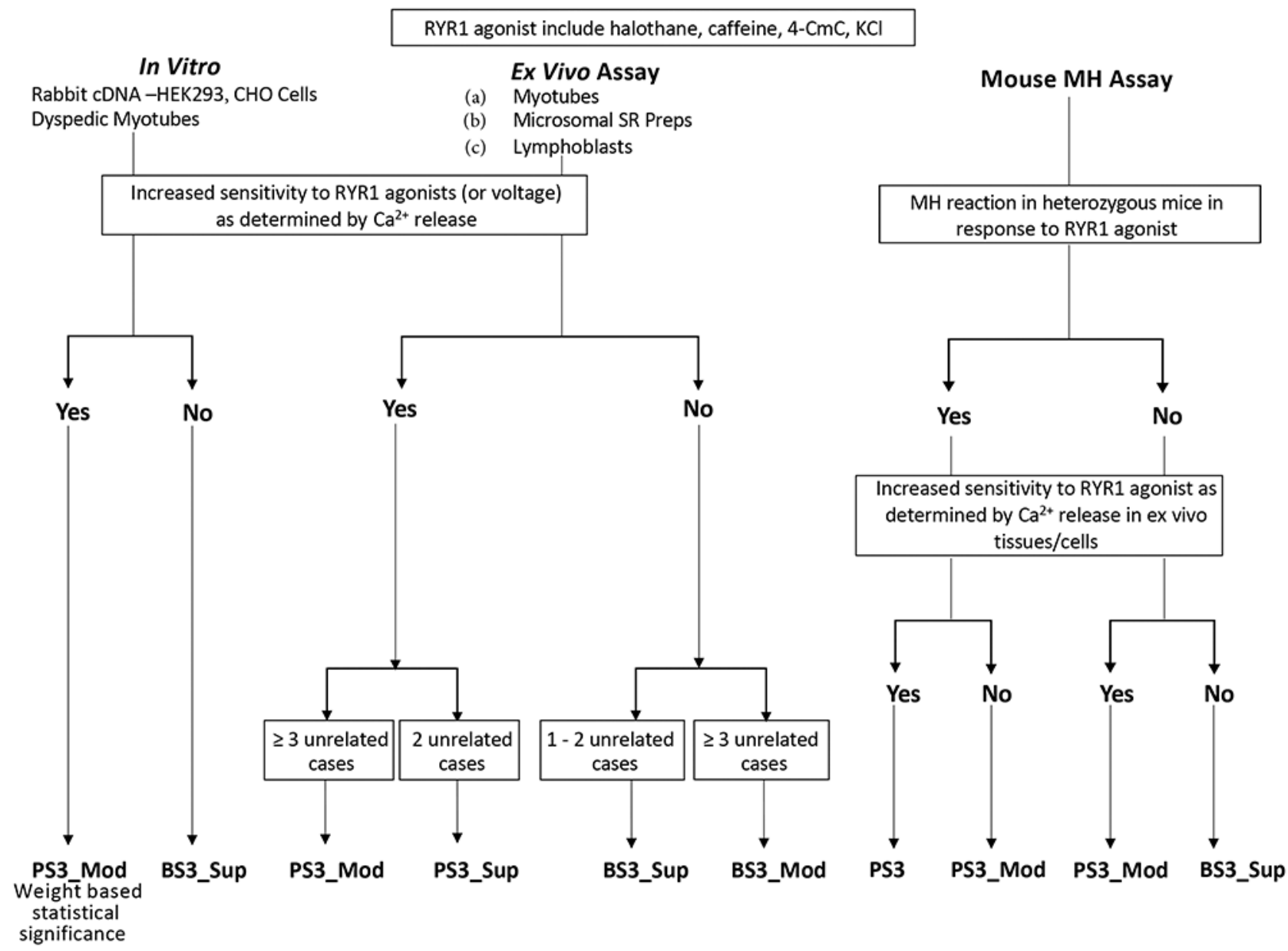

Figure 2.

Decision tree for weighting functional evidence PS3/BS3. 


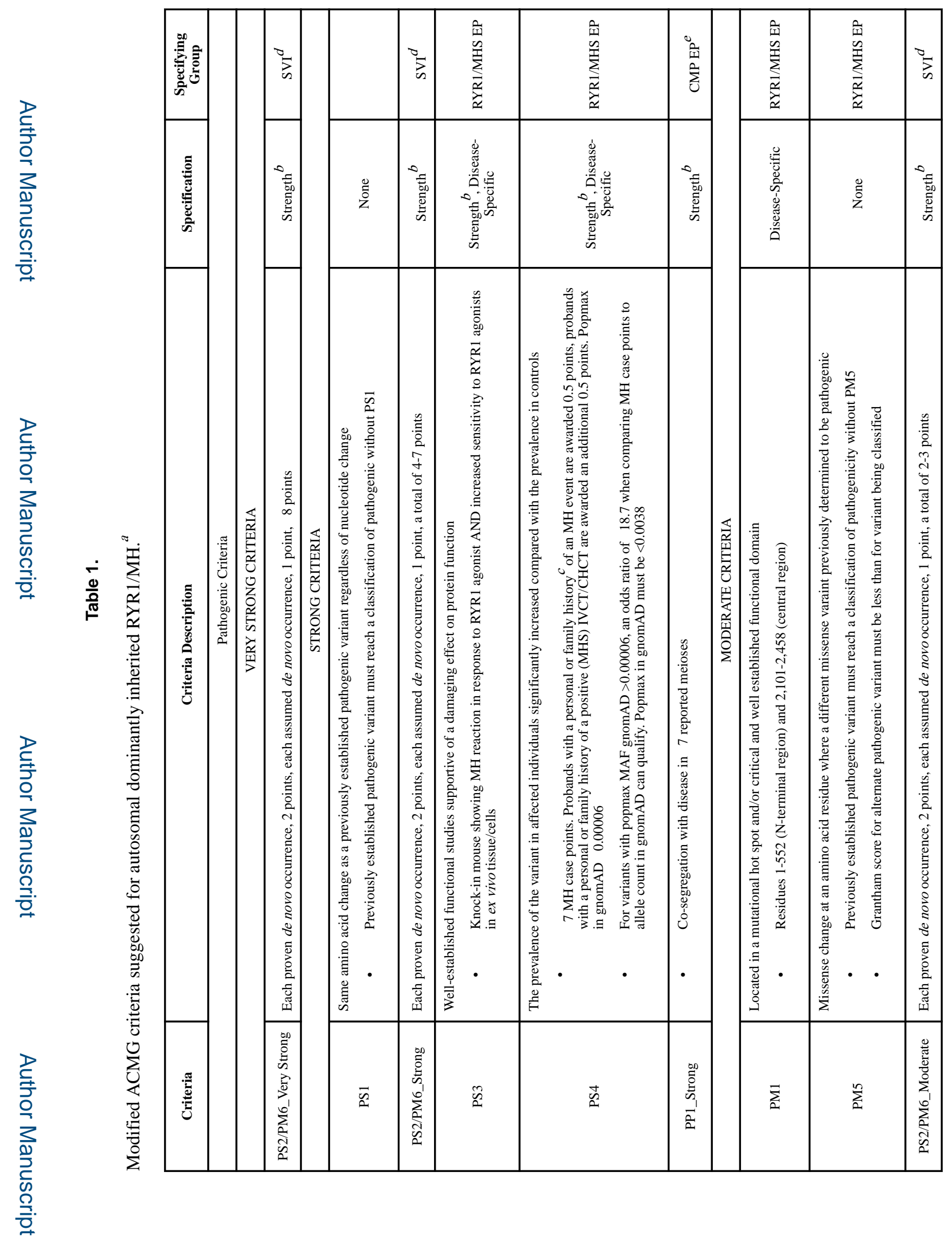

Genet Med. Author manuscript; available in PMC 2021 September 25. 


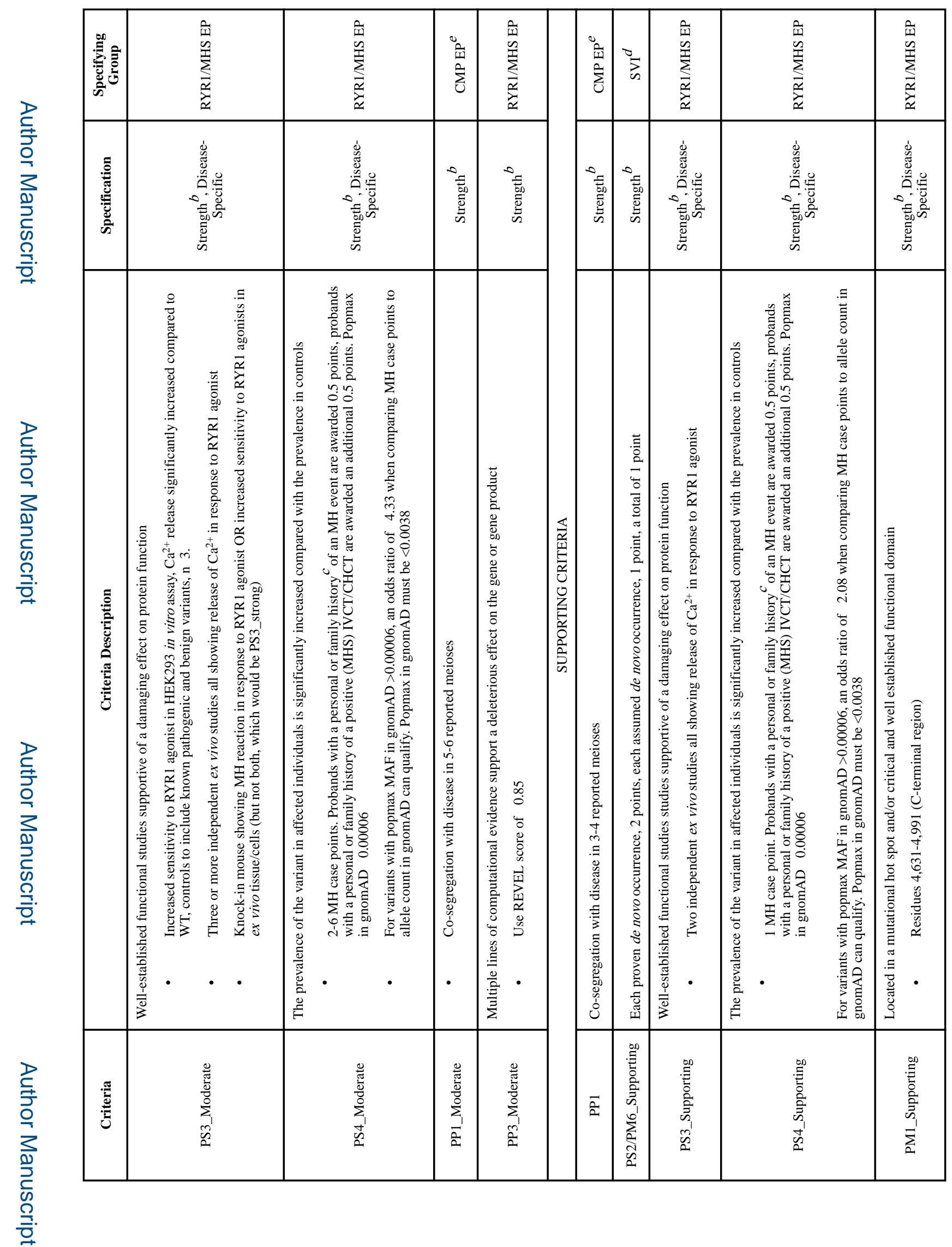

Genet Med. Author manuscript; available in PMC 2021 September 25. 


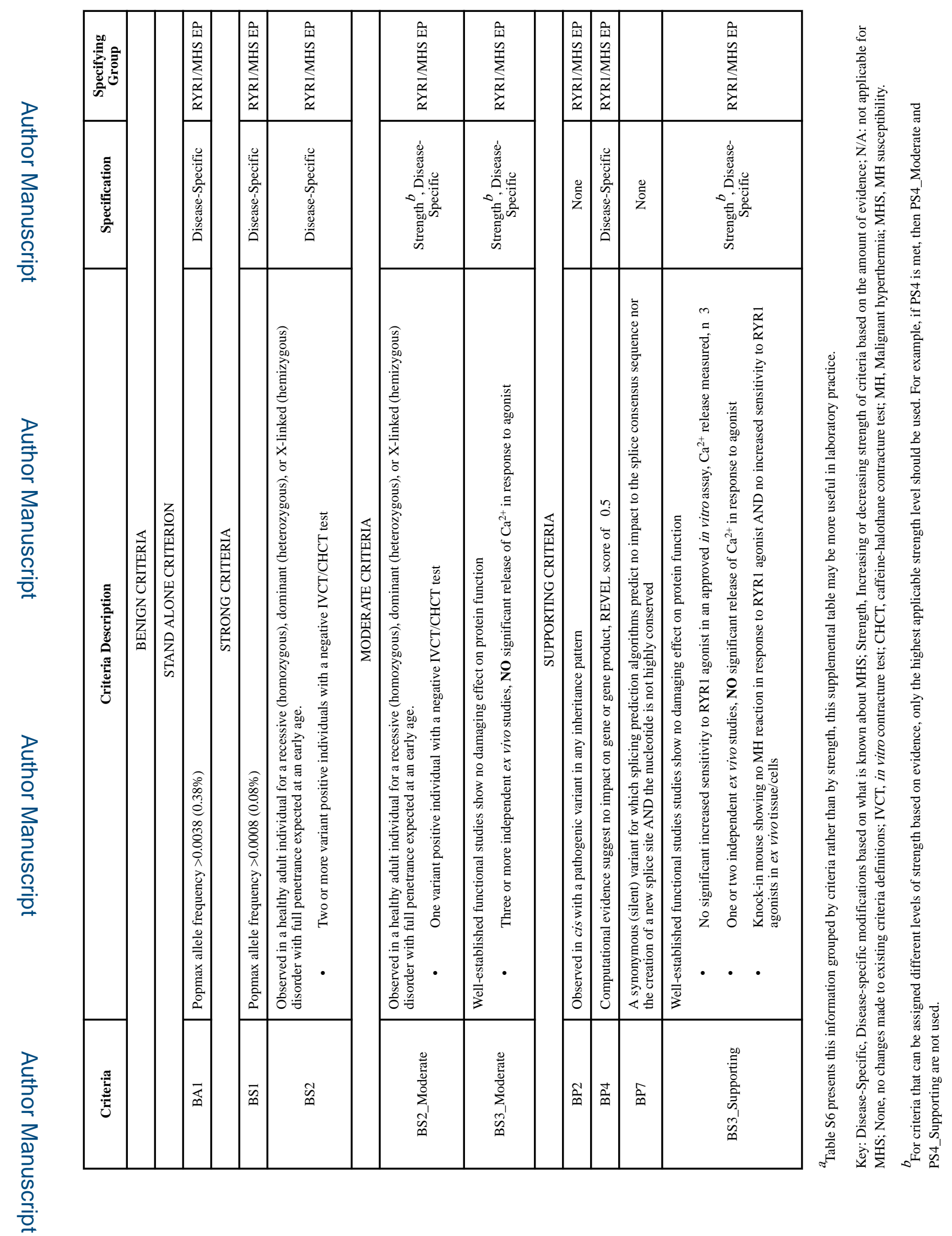

Genet Med. Author manuscript; available in PMC 2021 September 25. 

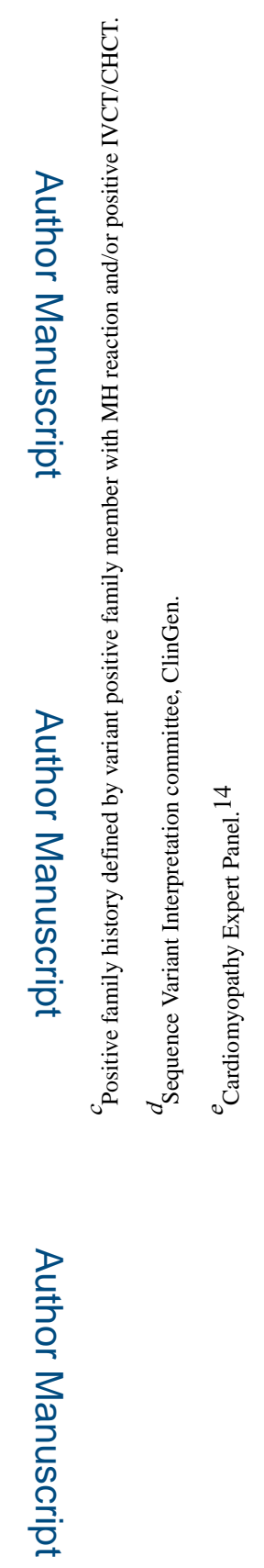

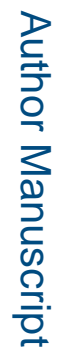

Genet Med. Author manuscript; available in PMC 2021 September 25. 
Table 2.

Distribution of 19 pathogenic and 27 benign variants in relation to position of defined $R Y R 1 / \mathrm{MH}$ hotspots. Likelihood ratios calculated based on distribution.

\begin{tabular}{|l|l|l|l|l|l|}
\hline $\begin{array}{l}\text { Presence in } \\
\text { HotSpot }\end{array}$ & Pathogenic & Benign & Likelihood ratio (LR) & Inverse LR & 95\% CI (inverse) \\
\hline HotSpot (1-552; 2101-2458; 4631-4991) & 16 & $1^{a}$ & 23.58 & & $3.41-163.18$ \\
\hline Non-HotSpot & 3 & 27 & 0.164 & 6.10 & $0.06-0.46(2.17-16.7)$ \\
\hline
\end{tabular}

${ }^{a}$ No benign variants were identified in the hotspot regions, for calculation of LR we used a value of 1 . 
Table 3.

REVEL score distribution for 20 pathogenic and 27 benign variants for $R Y R 1 / \mathrm{MH}$. Likelihood ratio for separation of pathogenic and benign variants based on REVEL scores using cutoff values of $\searrow 0.85$ and $₫) .5$.

\begin{tabular}{|l|l|l|l|l|l|}
\hline REVEL score & Pathogenic & Benign & $\begin{array}{l}\text { Likelihood } \\
\text { ratio (LR) }\end{array}$ & Inverse LR & 95\% CI \\
\hline$\searrow 0.85$ & 17 & $1^{a}$ & 22.68 & & $3.27-157.08$ \\
\hline$>0.5-<0.85$ & 3 & 8 & 0.50 & 2.00 & $0.15-1.66$ \\
\hline$\unlhd .5$ & $1^{a}$ & 19 & 0.07 & 14.29 & $0.01-0.48$ \\
\hline
\end{tabular}

${ }^{a}$ No benign variants were identified with a REVEL score $\searrow 0.85$ and no pathogenic variants were identified with a REVEL score $\left.₫\right) .5$, for calculation of LR we used a value of 1 . 